\title{
Metallic Icosahedron Phase of Sodium at Terapascal Pressures
}

\author{
Yinwei Li, ${ }^{1,2}$ Yanchao Wang, ${ }^{2}$ Chris J. Pickard, ${ }^{3}$ Richard J. Needs, ${ }^{4}$ Yi Wang, ${ }^{5}$ and Yanming Ma ${ }^{2, *}$ \\ ${ }^{1}$ School of Physics and Electronic Engineering, Jiangsu Normal University, Xuzhou 221116, China \\ ${ }^{2}$ State Key Laboratory of Superhard Materials, Jilin University, Changchun 130012, China \\ ${ }^{3}$ Department of Physics and Astronomy, University College London, Gower Street, London WC1E 6BT, United Kingdom \\ ${ }^{4}$ Theory of Condensed Matter Group, Cavendish Laboratory, J J Thomson Avenue, Cambridge CB3 OHE, United Kingdom \\ ${ }^{5}$ Natural Science Research Center, Academy of Fundamental and Interdisciplinary Sciences, \\ Harbin Institute of Technology, Harbin 150080, China
}

(Received 5 December 2014; published 23 March 2015)

\begin{abstract}
Alkali metals exhibit unexpected structures and electronic behavior at high pressures. Compression of metallic sodium (Na) to $200 \mathrm{GPa}$ leads to the stability of a wide-band-gap insulator with the double hexagonal $h P 4$ structure. Post- $h P 4$ structures remain unexplored, but they are important for addressing the question of the pressure at which Na reverts to a metal. Here, we report the reentrant metallicity of $\mathrm{Na}$ at the very high pressure of 15.5 terapascal (TPa), predicted using first-principles structure searching simulations. $\mathrm{Na}$ is therefore insulating over the large pressure range of 0.2-15.5 TPa. Unusually, Na adopts an $o P 8$ structure at pressures of 117-125 GPa and the same $o P 8$ structure at 1.75-15.5 TPa. The metallization of $\mathrm{Na}$ occurs on the formation of a stable and striking body-centered cubic cI24 electride structure consisting of $\mathrm{Na}_{12}$ icosahedra, each housing at its center about one electron that is not associated with any $\mathrm{Na}$ ions.
\end{abstract}

DOI: 10.1103/PhysRevLett.114.125501

Alkali metals have long been known to possess simple electronic structures at ambient pressure that are well explained by a nearly free-electron model. However, the simple low-pressure structures of alkali metals do not remain simple upon compression. Rich and complex phases and remarkable physical phenomena have been observed, such as greatly increased electrical resistivity [1], enhanced superconductivity $[2,3]$, unusually low melting temperatures $[4,5]$ and metal-insulator-semiconductor transitions [6,7].

The metal-insulator transitions in $\mathrm{Na}$ and lithium (Li) $[6,7]$ are among the most fascinating observations at high pressure. Neaton and Ashcroft predicted that upon compression $\mathrm{Li}$ [8] and $\mathrm{Na}$ [9] might transform into atomically paired structures. If a paired structure was to be adopted, both $\mathrm{Na}$ and $\mathrm{Li}$ would have the potential to be semiconducting. These predictions have stimulated numerous studies of the highpressure structures of $\mathrm{Li}$ and $\mathrm{Na}$ (e.g., Refs. [6,7,10-14]).

The metal-insulator transition in Na was established by a joint theoretical and experimental effort [7] and confirmed by further experiments [15]. Na is predicted and observed to transform into an optically transparent phase at above $200 \mathrm{GPa}$ [7]. This phase was predicted and experimentally confirmed to have a double hexagonal $h P 4$ structure [7], with a remarkably large band gap reaching $6.5 \mathrm{eV}$ at $600 \mathrm{GPa}$. A metal-semiconductor transition was observed in $\mathrm{Li}$ at $80 \mathrm{GPa}$. The semiconducting structure of dense $\mathrm{Li}$ remained unsolved despite considerable attention (e.g., Refs. [11-14]). Blind crystal structure prediction calculations [12] on dense Li predicted a complex semiconducting $o C 40$ structure. Independent experimental and theoretical studies also found that semiconducting $\mathrm{Li}$ adopts the $o C 40$ structure [16].
PACS numbers: 61.50.Ks, 61.50.Ah, 62.50.-p, 71.20.-b

Analysis of the charge density $[7,11,12]$ shows that the emergence of an insulating state in dense $\mathrm{Na} / \mathrm{Li}$ is, however, not attributable to atomic pairing, but rather to strong localization of valence electrons within lattice voids. Notably, the insulating phases of $\mathrm{Na}$ and $\mathrm{Li}$ can be regarded as prototypical examples of electrides [17], in which the ionic cores play the role of cations, and interstitial electrons form the anions $[7,11,12]$. Stable electride structures have also been predicted in other elements at high pressures, e.g., $\mathrm{Al}$ [18], $\mathrm{Mg}$ [19], $\mathrm{Ca}$ [20], and $\mathrm{K}$ [21]. The formation of an electride in $\mathrm{Na}$ at high pressures is favorable because it reduces the kinetic energy of the higher-energy electrons. These electronic orbitals are forced to oscillate rapidly close to the atomic cores due to Pauli repulsion, but if they move away from the cores the oscillations and, hence, the kinetic energy can be reduced $[17,18]$. The electrides have open structures if one considers only the ions, but when the interstitial electrons are considered as anions the structures make good chemical sense as ionic solids.

Pressure-induced metal-insulator transitions have been predicted in other materials at TPa pressures. For example, it was suggested that $\mathrm{Mg}$, a good metal at normal pressures, would transform from a metal to a semimetal at $24 \mathrm{TPa}$ [22], and $\mathrm{Ni}$ was predicted to transform to an insulator at $34 \mathrm{TPa}$ [23]. The metal-insulator transition in $\mathrm{Ni}$ is driven by the complete $4 s \rightarrow 3 d$ charge transfer under pressure, which is different from the electron localization found in $\mathrm{Li}$ and $\mathrm{Na}$.

Metallization is presumed to be the ultimate fate of all materials under sufficiently strong compression [24]. The insulating phase of $\mathrm{Ni}$ has been predicted to revert to metallic behavior at $51 \mathrm{TPa}$ [23]. Recent electrical resistance measurements have revealed that semiconducting $\mathrm{Li}$ 
reverts to a "poor metal" above $120 \mathrm{GPa}$ [10]. The question of the pressure at which $\mathrm{Na}$ will also revert to a metal remains unanswered. Determining the ground-state structures of $\mathrm{Na}$ at high pressures provides the key to whether insulating or metallic behavior is observed. At room temperature, $\mathrm{Na}$ undergoes pressure-induced phase transitions with an established sequence of $b c c \rightarrow f c c(65 \mathrm{GPa})$ [25] $\rightarrow$ $c I 16(103 \mathrm{GPa})$ [26-28] $\rightarrow$ oP8 (117 GPa) [26] $\rightarrow$ $t I 19(125 \mathrm{GPa})$ [26] $\rightarrow h P 4(200 \mathrm{GPa})$ [7]. The $h P 4$ structure is the highest-pressure phase of $\mathrm{Na}$ known so far.

In this Letter, we report the reentrant metallicity of $\mathrm{Na}$ at a surprisingly high pressure of $15.5 \mathrm{TPa}$, predicted via extensive structure searching in conjunction with firstprinciples calculations. We predict that $\mathrm{Na}$ will show insulating behavior over a remarkably large pressure range of $0.2-15.5 \mathrm{TPa}$ and will exhibit a phase transition to a second insulating orthorhombic oP8 structure at $1.75 \mathrm{TPa}$. Metallization of $\mathrm{Na}$ appears with the formation of a striking body-centered cubic (bcc) cI24 structure whose lattice sites are populated by $\mathrm{Na}_{12}$ icosahedra, each containing about one electron that is not associated with any $\mathrm{Na}$ ions.

Our structure searching calculations with simulation cells containing up to 24 atoms were performed in a wide pressure range (0.4-20 TPa). We used the efficient CALYPSO [29,30] and AIRSS [31,32] methods, which have both been successfully applied to investigating structures of materials at high pressures [12,31,33-42]. TPa pressures can now be achieved with dynamic ramped compression. In particular, diamond has been studied experimentally up to $5 \mathrm{TPa}$ [43]. The density functional theory [44] calculations were performed using the CASTEP plane-wave code [45] with the Perdew-Burke-Ernzerhof [46] (PBE) generalized gradient approximation functional. We performed calculations with both norm-conserving and ultrasoft $\mathrm{Na}$ pseudopotentials, in which only the $1 s$ energy level was treated as core. An ultrasoft pseudopotential was used for the structure searches, with a plane-wave cutoff energy of $910 \mathrm{eV}$ and a Brillouinzone integration grid spacing of $2 \pi \times 0.05 \AA^{-1}$. The structures obtained were reoptimized at a higher level of accuracy for both the norm-conserving and ultrasoft pseudopotentials. Here, we present only norm-conserving results since the two pseudopotentials led to consistent conclusions. A cutoff energy of $1633 \mathrm{eV}$ and a $k$-point grid spacing of $2 \pi \times$ $0.03 \AA^{-1}$ were used in the calculations with norm-conserving pseudopotentials. The phonon dispersion curves were computed by a finite displacement method as implemented in the CASTEP code.

Our structure searches readily reproduced the known double hexagonal $h P 4$ structure (space group $P 6_{3} / m m c$, 4 atoms/unit cell) above $400 \mathrm{GPa}$, and we found no better structure below 1.5 TPa. Surprisingly, above $2 \mathrm{TPa}$, we found the reappearance of the orthorhombic $o P 8$ structure [space group Pnma, 8 atoms/unit cell, Fig. 1(a)] which is also observed at low pressures of $117-125 \mathrm{GPa}$ [26].
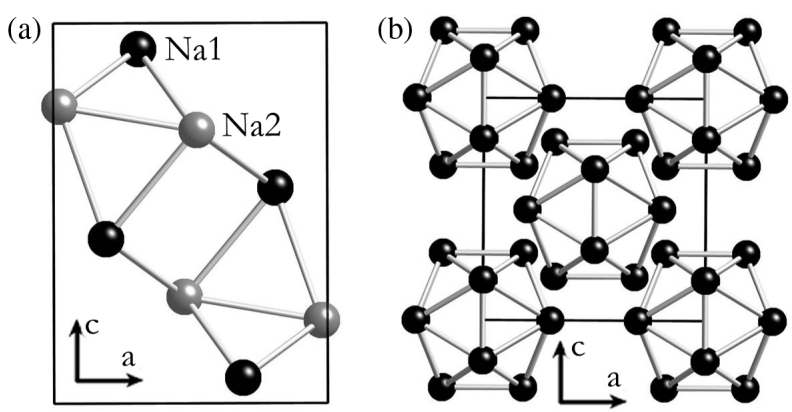

(c)
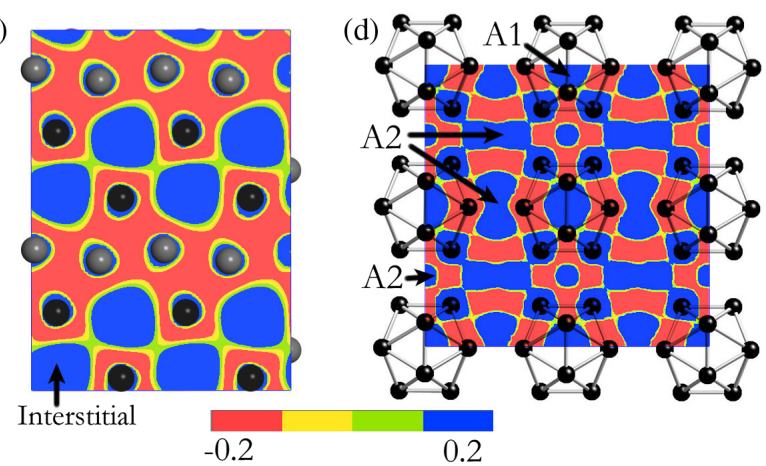

FIG. 1 (color online). Crystal structures of $o P 8$ (a) and $c I 24$ (b) and their electron density difference maps [(c) and (d)] at ultrahigh pressures. The lattice parameters of $o P 8-\mathrm{Na}$ at $2 \mathrm{TPa}$ are $a=2.88 \AA, b=2.12 \AA$, and $c=4.0 \AA$ with two inequivalent $\mathrm{Na} 1$ and $\mathrm{Na} 2$ atoms at $4 c$ positions of $(0.809,0.75,0.567)$ and $(0.523,0.25,0.719)$, respectively. For $c I 24-\mathrm{Na}$ at $16 \mathrm{TPa}$, $a=3.12 \AA$ with $\mathrm{Na}$ atoms at $24 g$ positions $(0,0.302,0.183)$. Electron density differences are plotted within the (010) plane of $o P 8-\mathrm{Na}$ (c) at $2 \mathrm{TPa}$ and $c I 24-\mathrm{Na}(\mathrm{d})$ at $16 \mathrm{TPa}$, respectively. $A 1$ and $A 2$ in (d) represent electron attractors located at the centers of the $\mathrm{Na}_{12}$ icosahedra and octahedral voids formed by the six neighboring $\mathrm{Na}_{12}$ icosahedra, respectively.

Strikingly, we predict a hitherto unexpected highly symmetric bcc structure at $20 \mathrm{TPa}$ [space group Im-3, 24 atoms/unit cell, denoted $c I 24$ hereafter, see Fig. 1(b)]. The $c I 24$ structure consists of $\mathrm{Na}_{12}$ cages, each of which contains $12 \mathrm{Na}$ atoms forming an icosahedron. The shortest intra- and intericosahedral $\mathrm{Na}-\mathrm{Na}$ distances at $16 \mathrm{TPa}$ are calculated to be 1.138 and $1.168 \AA$, respectively, indicating fairly strong core-core overlap (the ionic radius of $\mathrm{Na}^{+}$is $1.02 \AA)$. An analysis of electron density differences [Fig. 1(d)] reveals that the $c I 24$ phase is also an electride with two distinct electron attractors located at the centers of the $\mathrm{Na}_{12}$ icosahedra $(A 1)$ and the octahedral voids $(A 2)$. However, unlike the nearly spherical isosurface of the interstitial electrons in the $h P 4$ and $o P 8$ structures, dumbbell-like electron localization is found in the octahedral voids.

The enthalpies of the $o P 8$ (relative to $h P 4$ ) and $c I 24$ (relative to $o P 8$ ) phases are plotted as functions of pressure in Figs. 2(a) and 2(b), respectively. The inset to Fig. 2(a) shows that $h P 4-\mathrm{Na}$ transforms to the $o P 8$ structure at $1.75 \mathrm{TPa}$ with a continuous volume change, indicating a 

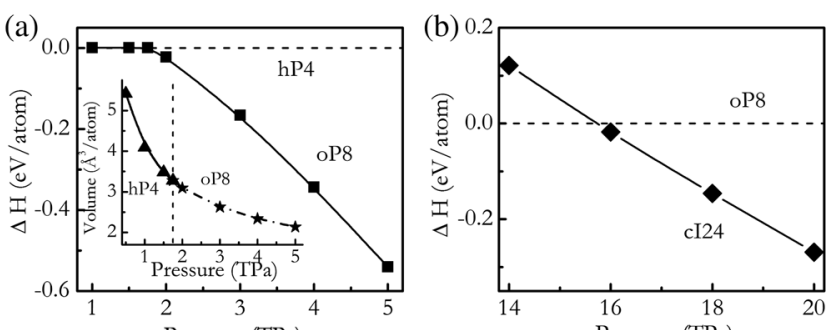

(c)
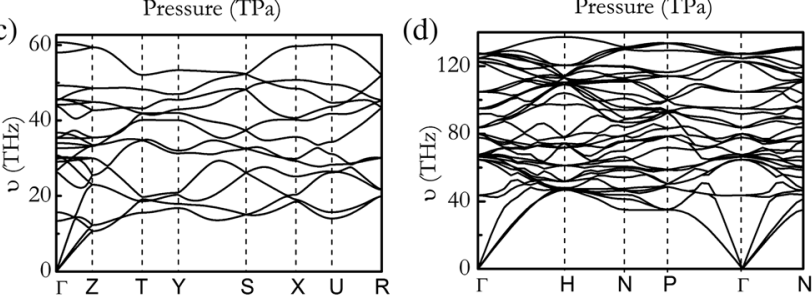

FIG. 2. Enthalpy differences relative to $h P 4$ (a) and $o P 8$ (b) of $\mathrm{Na}$ as a function of pressure. The inset in (a) shows the pressure dependence of the volumes of the $h P 4$ and $o P 8$ phases. The predicted sequence of phase transitions at zero temperature in the pressure range of $0-20 \mathrm{TPa}$ is provided in Fig. S1 of the Supplemental Material [47]. The volume appears to change continuously across the phase transition. [(c) and (d)] Calculated phonon dispersion relations of $o P 8-\mathrm{Na}$ at $2 \mathrm{TPa}$ and $c I 24-\mathrm{Na}$ at $16 \mathrm{TPa}$, respectively.

second-order phase transition. The $o P 8$ phase remains stable up to $15.5 \mathrm{TPa}$, above which the $c I 24$ phase becomes the most stable. Phonon calculations for $o P 8$ and $c I 24-\mathrm{Na}$ in their corresponding stable pressures ranges show no imaginary frequencies, demonstrating the dynamical stability of the structures [Figs. 2(c), 2(d)]. The maximum frequency in the TPa pressure regime reaches $137 \mathrm{THz}$ at $16 \mathrm{TPa}$, which is very high and implies a large nuclear zeropoint (ZP) energy. The estimated ZP energies within the quasiharmonic approximation are indeed extremely large: 0.577 and $0.585 \mathrm{eV} /$ atom for $c I 24$ and $o P 8$ phases at $20 \mathrm{TPa}$, respectively. However, the difference in $\mathrm{ZP}$ energies of only $8 \mathrm{meV} /$ atom is too small to modify the phase diagram of $\mathrm{Na}$ at $\mathrm{TPa}$ pressures. Furthermore, we have calculated the Gibbs free energies of the $c I 24$ and $o P 8$ phases at $20 \mathrm{TPa}$ within the quasiharmonic approximation at finite temperatures to account for vibrational contributions. The resultant difference in free energy between $\mathrm{cI} 24$ and $o P 8$ at $1000 \mathrm{~K}$ is only $3 \mathrm{meV} /$ atom larger than that at $0 \mathrm{~K}$ [Fig. 2(b)], which gives a negligible change in the oP8-cI24 transition pressure.

We calculated the phonon dispersion curves of $h P 4-\mathrm{Na}$ at various pressures in order to understand the origin of the reemergence of the $o P 8$ phase and the second-order nature of the $h P 4-o P 8$ transition. We found that a transverse acoustic (TA) phonon mode at the zone boundary $M \quad(00.50)$ point softens with increasing pressure [Fig. 3(a)], and its frequency goes to zero at $1.72 \mathrm{TPa}$ [Fig. 3(b)]. A frozen-phonon calculation was performed by distorting the $h P 4$ structure according to the atomic
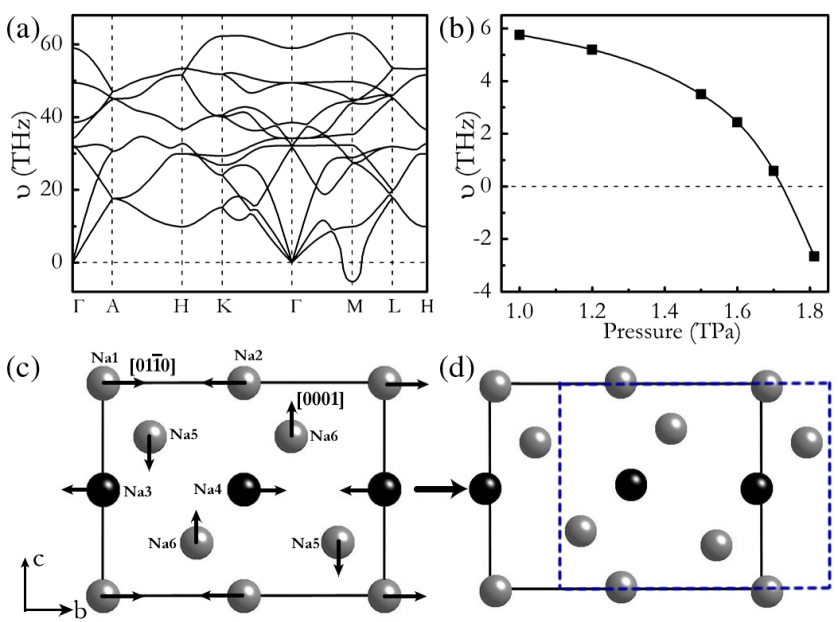

FIG. 3 (color online). Phonon dispersion curves of $h P 4-\mathrm{Na}$ at $2 \mathrm{TPa}$ (a) and the phonon frequencies of the softened TA phonon mode at $M(0,0.5,0)$ as a function of pressure (b). (c) Atomic vibrations of the soft TA mode at the $M$ point viewed along the $a$ axis in a $1 \times 2 \times 1$ supercell of the $h P 4$ structure. Here, the Na1 (Na2) and $\mathrm{Na} 4(\mathrm{Na} 3)$ atoms vibrate parallel (antiparallel) to the [0110] direction, while the $\mathrm{Na} 5(\mathrm{Na} 6)$ atoms vibrate antiparallel (parallel) to the [0001] direction. The gray and black balls indicate atoms in different planes. Arrows indicate vibrational direction of different $\mathrm{Na}$ atoms as labeled and are plotted from the eigenvector of the TA soft mode at the $M$ point. The blue dashed cell in (d) indicates the $o P 8$ structure resulting from the distortion.

vibrations for the TA mode at the $M$ point as depicted in Fig. 3(c), and structural optimizations were subsequently carried out. The resultant energetically stable structure is indeed the orthorhombic $o P 8$ phase, as indicated by the dashed cell in Fig. 3(d). It is clear that the $h P 4-o P 8$ transition is driven by the soft TA mode. This result is consistent with the second-order $h P 4-o P 8$ transition characterized by a continuous volume change [inset in Fig. 2(a)]. Soft-phonondriven $h P 4-o P 8$ transformations have also been reported in MnAs, CrTe, MnTe, MgTe, CrSb, and $\mathrm{CaH}_{2}$ [48-50].

The electronic band structures and band gaps of $h P 4$, $o P 8$, and $c I 24-\mathrm{Na}$ were calculated at selected pressures using the HSE06 functional [51,52], which is known to give better band gaps than the standard PBE functional. The band gap of $h P 4-\mathrm{Na}$ increases with pressure and reaches $9.5 \mathrm{eV}$ at the transition to the $o P 8$ phase at $1.75 \mathrm{TPa}$. At the transition, $o P 8-\mathrm{Na}$ is also a wide-band-gap insulator with an even larger gap of $9.7 \mathrm{eV}$ [Fig. 4(a)]. The gap of $o P 8-\mathrm{Na}$ decreases with pressure down to $1.6 \mathrm{eV}$ at $15.5 \mathrm{TPa}$. The $o P 8$ structure is compressed by a factor of 3 from $125 \mathrm{GPa}$ (volume $=77.9 \AA^{3}$ per unit cell) to 1.75 TPa (volume $=25.9 \AA^{3}$ per unit cell). As a result of this strong compression, the $o P 8$ structure at $\mathrm{TPa}$ pressures forms an electride with valence electrons trapped at the interstitial sites [Fig. 1(c)]. The electronic structure is fundamentally different from that of the nearly freeelectron behavior of $o P 8$ at a much lower pressure of 

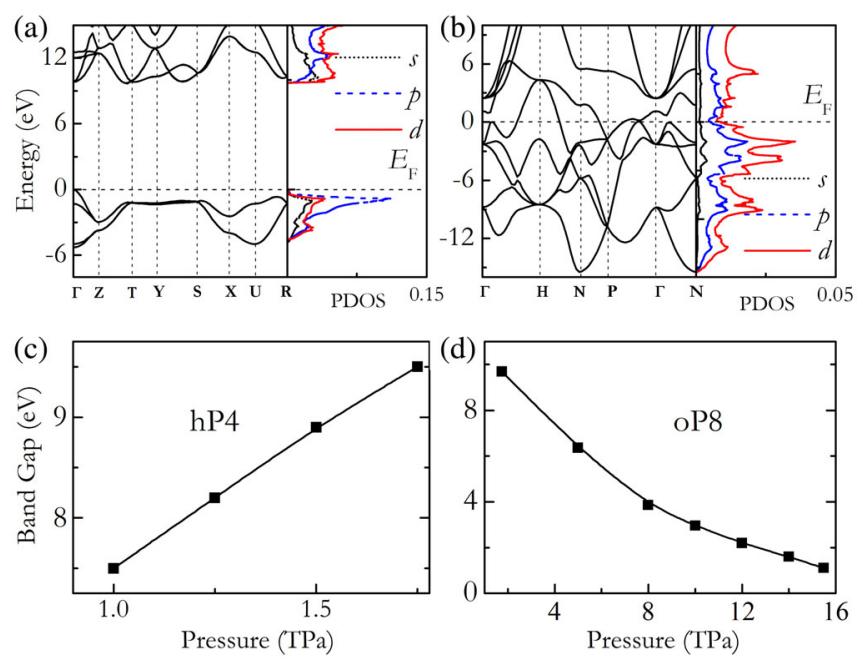

FIG. 4 (color online). Electronic band structure and projected density of states of $o P 8-\mathrm{Na}$ at 1.75 (PDOS, in units of $\mathrm{eV}^{-1}$ per atom) (a) and $c I 24-\mathrm{Na}$ at $16 \mathrm{TPa}(\mathrm{b})$. The horizontal dotted lines indicate the Fermi level. [(c) and (d)] Variation of band gaps with pressure for $h P 4$ and $o P 8-\mathrm{Na}$, respectively, using the HSE06 functional.

$125 \mathrm{GPa}$ (Supplemental Material Fig. S2 [47]). As a result, the $o P 8$ phase is insulating at TPa pressures but is metallic at pressure below $125 \mathrm{GPa}[26,47]$.

After the transition to the $c I 24$ phase, the localized electrons in the octahedral voids are partially squeezed out due to the decreasing interstitial space, forming a series of conducting channels between $A 2$ attractors and $\mathrm{Na}$ cations, as shown in Fig. 1(d). Notably, the band structure of $c I 24-$ $\mathrm{Na}$ at $16 \mathrm{TPa}$ shows highly dispersive bands crossing the Fermi level [Fig. 4(b)] and the return to metallicity.

The enthalpy $H=U+P V$ governs the phase stability at $0 \mathrm{~K}$. Under sufficiently strong compression, the hard core repulsion between atoms becomes very important, and it favors close-packed structures such as (bcc, $f c c$ or $h c p)$ [53], which have small volumes $V$. However, the $c I 24$ structure that $\mathrm{Na}$ adopts at TPa pressure is far from simple and is constructed from $\mathrm{Na}_{12}$ icosahedral cages, further challenging the established picture of simple packing at high densities. Indeed, our optimized hypothetical $b c c$ structure with $\mathrm{Na}$ atoms occupying the lattice sites at $20 \mathrm{TPa}$ gives a volume of $1.175 \AA^{3} /$ atom, which has a lower density than $c I 24-\mathrm{Na}\left(1.148 \AA^{3} /\right.$ atom) whose $b c c$ lattice sites are occupied by $\mathrm{Na}_{12}$ icosahedral cages. Integration of the electron density at $16 \mathrm{TPa}$ shows that the charge on each $\mathrm{Na}$ atom is depleted by about $\sim 0.35$ electrons, while the cage-center $A 1$ and interstitial $A 2$ sites [Fig. 1(d)] have excess electronic charges of about $\sim 1.2$ and $\sim 1.0$ electrons, respectively. As a whole, 9 of the 12 valence electrons remain within each $\mathrm{Na}_{12}$ cage (including the localized central electrons), while 3 electrons localize in the octahedral voids. The 9-electron configuration might allow a $\mathrm{Na}_{12}$ cage to be termed a superalkali atom.
Electronically, a $\mathrm{Na}_{12}$ cage resembles a potassium atom with an electronic configuration of $3 s^{2} 3 p^{6} 4 s^{1}$.

Cagelike structures are seldom seen at high pressures since they do not usually favor dense packing. Known cage structures are typically found in elements in which it is easy to form covalent bonds. Examples occur in various allotropes of $\mathrm{B}\left(\alpha-\mathrm{B}_{12}[54]\right.$ and $\gamma-\mathrm{B}_{28}$ [55]) and $\mathrm{C}\left(\mathrm{C}_{60}\right.$ and $\left.\mathrm{C}_{70}\right)$ [56] and $\mathrm{Si} / \mathrm{Ge}$ cages in clathrates (e.g., in $\mathrm{Ba}_{8} \mathrm{Si}_{46}$ [57]). In recent work, we also found a $\mathrm{N}_{10}$ cage structure in polymeric nitrogen [58] and a $\mathrm{H}_{24}$ cage in hydrogen-rich $\mathrm{CaH}_{6}$ [37]. Here, the formation of $\mathrm{Na}_{12}$ icosahedra is rather surprising since $\mathrm{Na}$ is unable to stabilize covalent bonds. The $\mathrm{Na}_{12}$ cage is geometrically similar to a $\mathrm{B}_{12}$ cage, which is the fundamental building block of various boron allotropes [54,55]. B is electron deficient and forms multicenter covalent bonds. Three-center covalent bonds within the icosahedra and two- or three-center covalent bonds between the icosahedra in various structures of elemental $\mathrm{B}$ satisfy the octet rule and generate insulating states. Here, there is no covalent bonding within the $\mathrm{Na}_{12}$ cages, and it is actually a well-packed ionic structure. The electrons at the center of the cage contribute to the stability of the $\mathrm{Na}_{12}$ cages and play a role similar to that of $\mathrm{Ba}$ atoms in the $\mathrm{Ba}_{8} \mathrm{Si}_{46}$ clathrate structure [57].

We find that $c I 24-\mathrm{Na}$ is structurally similar to the $b c c$ form of the $\mathrm{Al}_{12} \mathrm{~W}$ alloy [59], in which $\mathrm{Al}_{12}$ also forms an icosahedral cage with a $\mathrm{W}$ atom localized at its center, and the W-encaged $\mathrm{Al}_{12}$ icosahedrons occupy bcc lattice sites. One $\mathrm{Al}_{12} \mathrm{~W}$ unit resembles a $\mathrm{Na}_{12}$ icosahedron, and the encaged electron plays the role of the pseudoions in W. In contrast to the single dumbbell-like electron attractor found in the octahedral voids of $c I 24-\mathrm{Na}$, the voids in $\mathrm{Al}_{12} \mathrm{~W}$ contain four distinct dumbbell-like electron attractors (Supplemental Material Fig. S5 [47]).

$\mathrm{Na}$ is insulating over the large pressure range of 0.2 15.5 TPa. The pressure interval of $15.3 \mathrm{TPa}(98.7 \%$ of the pressure range $0-15.5 \mathrm{TPa}$ ) is comparable to the $17 \mathrm{TPa}$ (33.3\% of the whole pressure range) pressure interval predicted for the insulating phase of $\mathrm{Ni}$ [23]. The pressure for reentrant metallicity in Na (15.5 TPa) is much higher than that in $\mathrm{Li}(120 \mathrm{GPa})$, which may be associated with the entirely different mechanism for insulating behavior. In the $h P 4$ and $o P 8$ structures of $\mathrm{Na}$, the valence electrons localize strongly within the lattice interstices and maintain a nearly spherical shape [Fig. 1(c)] up to $15.5 \mathrm{TPa}$. However, semiconducting Li has a complex $o C 40$ structure with interstitial regions of different shapes in which electrons are localized at a rather low pressure of $80 \mathrm{GPa}$. These localized electrons are connected to each other and form conducting channels at $120 \mathrm{GPa}$ (Supplemental Material [47]).

Y.L., Y.W., and Y.M. acknowledge funding from the China 973 Program under Grant No. 2011CB808200 and the National Natural Science Foundation of China under Grants No. 11274136, No. 11025418, and No. 91022029. 
Y. L. also acknowledges funding from the National Natural Science Foundation of China under Grants No. 11204111 and No. 11404148, the Natural Science Foundation of Jiangsu province under Grant No. BK20130223, and the PAPD of Jiangsu Higher Education Institutions. C. J. P. and R. J. N. acknowledge funding from the Engineering and Physical Sciences Research Council of the U.K.

*mym@jlu.edu.cn

[1] V. E. Fortov, V. V. Yakushev, K. L. Kagan, I. V. Lomonosov, V. I. Postnov, and T. I. Yakusheva, JETP Lett. 70, 628 (1999).

[2] J. Wittig, Phys. Rev. Lett. 24, 812 (1970).

[3] V. V. Struzhkin, M. I. Eremets, W. Gan, H. K. Mao, and R. J. Hemley, Science 298, 1213 (2002).

[4] C.-S. Zha and R. Boehler, Phys. Rev. B 31, 3199 (1985).

[5] E. Gregoryanz, O. Degtyareva, M. Somayazulu, R. J. Hemley, and H.-k. Mao, Phys. Rev. Lett. 94, 185502 (2005).

[6] T. Matsuoka and K. Shimizu, Nature (London) 458, 186 (2009).

[7] Y. Ma, M. Eremets, A. R. Oganov, Y. Xie, I. Trojan, S. Medvedev, A. O. Lyakhov, M. Valle, and V. Prakapenka, Nature (London) 458, 182 (2009).

[8] J. B. Neaton and N. W. Ashcroft, Nature (London) 400, 141 (1999).

[9] J. B. Neaton and N. W. Ashcroft, Phys. Rev. Lett. 86, 2830 (2001).

[10] T. Matsuoka, M. Sakata, Y. Nakamoto, K. Takahama, K. Ichimaru, K. Mukai, K. Ohta, N. Hirao, Y. Ohishi, and K. Shimizu, Phys. Rev. B 89, 144103 (2014).

[11] M. Marqués, M. I. McMahon, E. Gregoryanz, M. Hanfland, C. L. Guillaume, C. J. Pickard, G. J. Ackland, and R. J. Nelmes, Phys. Rev. Lett. 106, 095502 (2011).

[12] J. Lv, Y. Wang, L. Zhu, and Y. Ma, Phys. Rev. Lett. 106, 015503 (2011).

[13] Y. Yao, J. S. Tse, and D. D. Klug, Phys. Rev. Lett. 102, 115503 (2009).

[14] C. J. Pickard and R. J. Needs, Phys. Rev. Lett. 102, 146401 (2009).

[15] M. Marqués, M. Santoro, C. L. Guillaume, F. A. Gorelli, J. Contreras-García, R. T. Howie, A. F. Goncharov, and E. Gregoryanz, Phys. Rev. B 83, 184106 (2011).

[16] C. L. Guillaume, E. Gregoryanz, O. Degtyareva, M. I. McMahon, M. Hanfland, S. Evans, M. Guthrie, S. V. Sinogeikin, and H. K. Mao, Nat. Phys. 7, 211 (2011).

[17] M.-S. Miao and R. Hoffmann, Acc. Chem. Res. 47, 1311 (2014).

[18] C. J. Pickard and R. Needs, Nat. Mater. 9, 624 (2010).

[19] P. Li, G. Gao, Y. Wang, and Y. Ma, J. Phys. Chem. C 114, 21745 (2010).

[20] A. R. Oganov, Y. Ma, Y. Xu, I. Errea, A. Bergara, and A. O. Lyakhov, Proc. Natl. Acad. Sci. U.S.A. 107, 7646 (2010).

[21] C. J. Pickard and R. J. Needs, Phys. Rev. Lett. 107, 087201 (2011).

[22] J. A. Moriarty and A. K. McMahan, Phys. Rev. Lett. 48, 809 (1982).

[23] A. K. McMahan and R. C. Albers, Phys. Rev. Lett. 49, 1198 (1982).

[24] E. Wigner and H. Huntington, J. Chem. Phys. 3, 764 (1935).
[25] M. Hanfland, I. Loa, and K. Syassen, Phys. Rev. B 65, 184109 (2002).

[26] E. Gregoryanz, L. F. Lundegaard, M. I. McMahon, C. Guillaume, R. J. Nelmes, and M. Mezouar, Science 320, 1054 (2008).

[27] M. I. McMahon, E. Gregoryanz, L. F. Lundegaard, I. Loa, C. Guillaume, R. J. Nelmes, A. K. Kleppe, M. Amboage, H. Wilhelm, and A. P. Jephcoat, Proc. Natl. Acad. Sci. U.S.A. 104, 17297 (2007).

[28] M. Hanfland, K. Syassen, L. Loa, N. E. Christensen, and D. L. Novikov, Poster at 2002 High Pressure Gordon Conference, 2002.

[29] Y. Wang, J. Lv, L. Zhu, and Y. Ma, Phys. Rev. B 82, 094116 (2010).

[30] Y. Wang, J. Lv, L. Zhu, and Y. Ma, Comput. Phys. Commun. 183, 2063 (2012).

[31] C. J. Pickard and R. J. Needs, Phys. Rev. Lett. 97, 045504 (2006).

[32] C. J. Pickard and R. J. Needs, J. Phys. Condens. Matter 23, 053201 (2011).

[33] Q. Li, D. Zhou, W. Zheng, Y. Ma, and C. Chen, Phys. Rev. Lett. 110, 136403 (2013).

[34] L. Zhu, Z. Wang, Y. Wang, G. Zou, H. Mao, and Y. Ma, Proc. Natl. Acad. Sci. U.S.A. 109, 751 (2011).

[35] L. Zhu, H. Wang, Y. Wang, J. Lv, Y. Ma, Q. Cui, Y. Ma, and G. Zou, Phys. Rev. Lett. 106, 145501 (2011).

[36] Y. Wang, H. Liu, J. Lv, L. Zhu, H. Wang, and Y. Ma, Nat. Commun. 2, 563 (2011).

[37] H. Wang, S. T. John, K. Tanaka, T. Iitaka, and Y. Ma, Proc. Natl. Acad. Sci. U.S.A. 109, 6463 (2012).

[38] C. J. Pickard and R. J. Needs, J. Chem. Phys. 127, 244503 (2007).

[39] C. J. Pickard and R. J. Needs, Nat. Phys. 3, 473 (2007).

[40] J. M. McMahon and D. M. Ceperley, Phys. Rev. Lett. 106, 165302 (2011).

[41] C. J. Pickard, M. Martinez-Canales, and R. J. Needs, Phys. Rev. B 85, 214114 (2012).

[42] J. Sun, M. Martinez-Canales, D. D. Klug, C. J. Pickard, and R. J. Needs, Phys. Rev. Lett. 108, 045503 (2012).

[43] R. Smith, J. Eggert, R. Jeanloz, T. Duffy, D. Braun, J. Patterson, R. Rudd, J. Biener, A. Lazicki, and A. Hamza, Nature (London) 511, 330 (2014).

[44] S. Baroni, P. Giannozzi, and A. Testa, Phys. Rev. Lett. 58, 1861 (1987).

[45] S. J. Clark, M. D. Segall, C. J. Pickard, P. J. Hasnip, M. I. Probert, K. Refson, and M. C. Payne, Z. Kristallogr. 220, 567 (2005).

[46] J. P. Perdew, K. Burke, and M. Ernzerhof, Phys. Rev. Lett. 77, 3865 (1996).

[47] See the Supplemental Material at http://link.aps.org/ supplemental/10.1103/PhysRevLett.114.125501 for phase sequence, band structures, density of states, and electron density difference.

[48] Y. W. Li, B. Li, T. Cui, Y. Li, L. J. Zhang, Y. M. Ma, and G. T. Zou, J. Phys. Condens. Matter 20, 045211 (2008).

[49] Y. Li, Y. Ma, T. Cui, Y. Yan, and G. Zou, Appl. Phys. Lett. 92, 101907 (2008).

[50] Y. Li, Y. W. Li, Y. Ma, T. Cui, and G. Zou, Phys. Rev. B 81, 052101 (2010). 
[51] J. Heyd, G. E. Scuseria, and M. Ernzerhof, J. Chem. Phys. 118, 8207 (2003).

[52] A. V. Krukau, O. A. Vydrov, A. F. Izmaylov, and G. E. Scuseria, J. Chem. Phys. 125, 224106 (2006).

[53] B. Rousseau, Y. Xie, Y. Ma, and A. Bergara, Eur. Phys. J. B 81, 1 (2011).

[54] A. Masago, K. Shirai, and H. Katayama-Yoshida, Phys. Rev. B 73, 104102 (2006).

[55] A. R. Oganov, J. Chen, C. Gatti, Y. Ma, C. W. Glass, Z. Liu, T. Yu, O. O. Kurakevych, and V. L. Solozhenko, Nature (London) 457, 863 (2009).
[56] W. Krätschmer, L. D. Lamb, K. Fostiropoulos, and D. R. Huffman, Nature (London) 347, 354 (1990).

[57] S. Yamanaka, E. Enishi, H. Fukuoka, and M. Yasukawa, Inorg. Chem. 39, 56 (2000).

[58] X. Wang, Y. Wang, M. Miao, X. Zhong, J. Lv, T. Cui, J. Li, L. Chen, C. J. Pickard, and Y. Ma, Phys. Rev. Lett. 109, 175502 (2012).

[59] W. B. Pearson, Crystal Chemistry and Physics of Metals and Alloys (Wiley, New York, 1972). 